\section{Unilateral demodicidosis of face mimicking Hansens disease}

\author{
Deepak Vashisht, ${ }^{1}$ Jatinder Singh, ${ }^{1}$ \\ Sukriti Baveja, ${ }^{1}$ Rohit Tiwari, ${ }^{2}$ \\ Anuj Bhatnagar ${ }^{1}$ \\ Departments of ${ }^{1}$ Dermatology and \\ 2Pathology, Armed Forces Medical \\ College, Pune, India
}

\begin{abstract}
Demodicosis is a common parasitic infection of the hair follicles and the pilosebaceous unit by the Demodex mites viz. Demodex folliculorum and Demodex brevis. Infection by this parasite is common among immunocompromised and elderly. We report a case of facial Demodicosis which presented like atypical rosacea with a gradually progressing swelling and redness on right side of face which was initially diagnosed as a case of Hansen's disease. Skin biopsy revealed follicular dilatation with presence of Demodex mite along with intense perifollicular lymphomononuclear infiltrate. Patient was treated with oral tab Ivermectin $12 \mathrm{mg}$ stat along with topical gel metronidazole twice daily to which he responded favourably.
\end{abstract}

\section{Introduction}

Mite Demodex is an ectoparasite infesting the areas of face rich in pilo-sebaceous units and less commonly other seborrheic sites like upper and medial region of chest and back are also involved. ${ }^{1,2}$ Majority of the patients are immune-compromised though immunocompetent are not spared, nevertheless atypical presention in immunocompetent individuals is a rarity. We report a case with atypical presentation which posed diagnostic dilemma due to confusing clinical picture.

\section{Case Report}

A 32-year-old male presented with gradual onset swelling and redness on right side of face associated with minimal itching for past six months. It started as a pea-sized red raised flat lesion, which increased in size to involve $1 / 3$ rd of right cheek, adjoining nasolabial fold and lower eye-lid. There was modest increase in redness and pruritus of the lesions on sunexposure, however hot spicy meals, alcohol or emotional stress did not aggravate his symp- toms. There was no history of cough, chest pain, fever, weight loss or any constitutional or systemic symptoms. Patient denied using any local or systemic medication. There was no history of pets in the house. Patient reported to a dermatologist about a month back, where he was diagnosed as Hansen's disease in view of equivocal hypoaesthesia and solitary indurated plaque on face. He underwent skin biopsy which revealed non-specific inflammation. He was empirically started on multi drug treatment (MDT) multi bacillary for Hansens disease. After about four weeks of MDT, patient reported to our hospital with no clinical improvement and persistence of symptoms. His general physical and systemic examinations were within normal limits. Dermatological examination revealed involvement of right malar region, nasolabial fold and adjacent parts of nasal bridge and lower eyelid in the form of edema, erythema and follicular dilatation (Figure 1). There was no hypoesthesia or thickened peripheral nerves. Laboratory investigations revealed complete blood count, erythrocyte sedimentation rate (ESR), liver and renal function tests, serum ACE levels, were normal. Screening for hepatitis B, hepatitis $\mathrm{C}$ and human immunodeficiency virus, as well as serology for antinuclear antibodies (ANA) were negative. Chest X-ray showed no abnormality and $\mathrm{KOH}$ mount of scrapings did not reveal any pathology. Repeat skin biopsy from medial end of the swelling along nasolabial fold showed mild epidermal orthohyperkeratosis. The dermis showed intense follicular and perifollicular lymphocytic inflammatory infiltrate along with dense lichenoid lymphomononuclear infiltrate. There was follicular dilatation with presence of Demodex sp.mite with surrounding homogenous eosinophilic material (Figure 2).

Patient was managed with oral ivermectin $12 \mathrm{mg}$ stat and topical metronidazole gel locally twice a day. The eryhthema and swelling started regressing within few days and after 03 weeks had near complete resolution of symptoms (Figure 3).
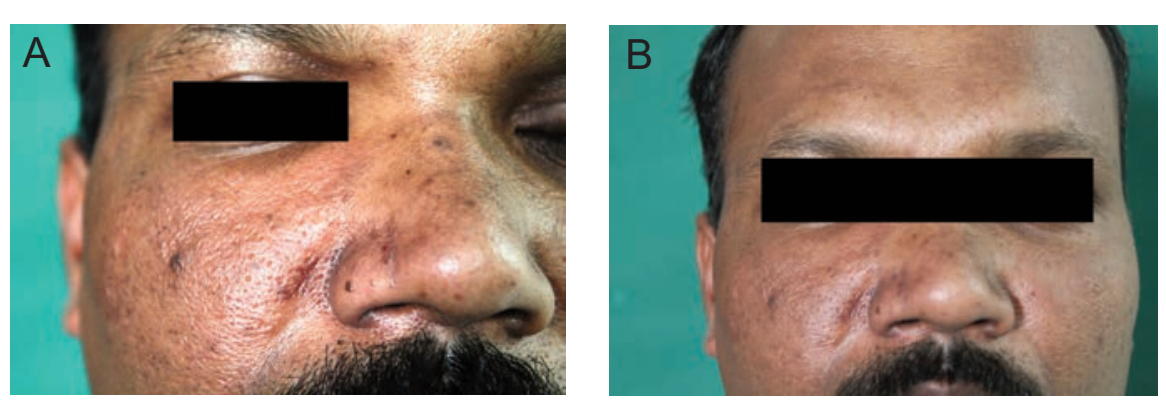

Figure 1. A) Partially defined edematous and erythematous plaque on right malar region, nasolabial fold and lower eyelid with follicular dilatation. B) Comparisson of the lesion with contralateral normal site.

Correspondence: Deepak Vashisht, Department of Dermatology, Armed Forces Medical College, Pune, Maharasthra, 411040 India.

Tel.: +91.9419021643.

E-mail: deepak3975@gmail.com

Key words: Demodicosis, atypical rosacea, Hansen's Disease.

Contributions: the authors contributed equally.

Conflict of interest: the authors declare no potential conflict of interest.

Received for publication: 16 September 2016. Accepted for publication: 18 November 2016.

This work is licensed under a Creative Commons Attribution-NonCommercial 4.0 International License (CC BY-NC 4.0).

(C) Copyright D. Vashisht et al., 2016

Licensee PAGEPress, Italy

Dermatology Reports 2016; 8:6891

doi:10.4081/dr.2016.6891

\section{Discussion and Conclusions}

Demodex are parasitic mites that live in hair follicles and pilosebaceous units, accordingly maximum density is found in seborrheic sites. Besides humans they also cause significant infestation in canines and felines. ${ }^{3}$ Human infestation is very common and varies between $23-100 \%$ as per different authors. ${ }^{4,5}$ This infestation is usually asymptomatic, but when and how it becomes pathogenic is poorly understood. ${ }^{6}$ Primary or secondary suppression in immunity plays a major role in Demodex proliferation and resultant dermatoses. Cutaneous disorders attributed to this mite include, rosacea, pustular folliculitis, perioral dermatitis, Lupus miliaris disseminatus faciei, Madarosis, non-specific facial dermatitis etc. ${ }^{7}$ Our patient presented with nonspecific facial dermatitis affecting only right 


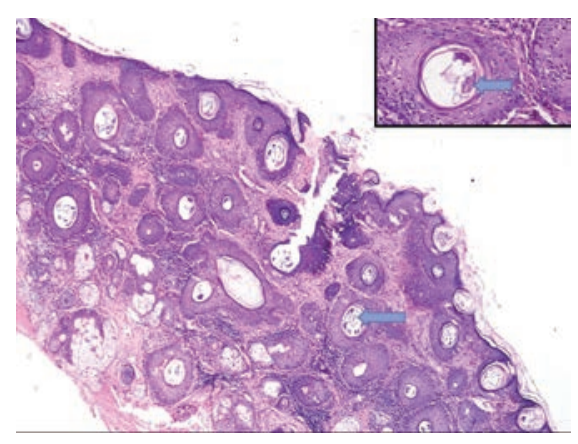

Figure 2. Section through skin biopsy showing multiple follicular infundibula showing Demodex mites (horizontal arrow). The perifollicular tissue shows lymphomononuclear inflammation (H\&E, 100 $)$. Inset: Demodex mite in the infundibulum(horizontal arrow) (H\&E, $400 \times)$.

side of the face. Patient had no evidence of primary or secondary immune suppression. There was no local or systemic risk factor suggestive of Demodex infestation. Unilateral, indurated, erythematous plaque on face with equivocal hypoaesthesia resulted in initial misdiagnoses. Earlier it has also been reported to present with facial sclera-oedema and ble-

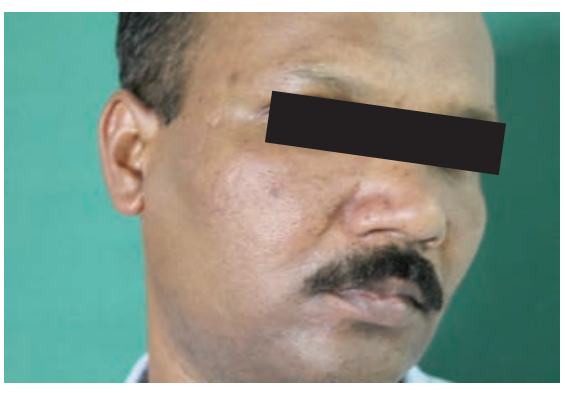

Figure 3. Almost completed resolution of the plaque on right malar region.

pharoedema which mimicked cutaneous lymphoma. ${ }^{8}$ This case is being reported for its unilateral atypical presentation. Further demodicidosis should also be considered in patients presenting even with unilateral lesions in seborrheic areas.

\section{References}

1. Aylesworth R, Vance C.Demodex folliculorum and Demodex brevis in cutaneous biopsies. J Am Acad Dermatol 1982;7:583-9.
2. Basta- Juzbasic A, Subic JS, Ljubojevic S. Demodex folliculorum in development of dermatitis rosaceiformis steroidica and rosacea-related diseases. Clin Dermatol 2002;20:135-40.

3. Gross TL, Ihrke PJ, Walder EJ, Affolter VK. Skin diseases of the dog and cat: clinical and histopathologic diagnosis. 0xford: Blackwell Science Ltd; 2008. pp 932

4. Norn MS. Demodex folliculorum. Incidence, regional distribution, pathogenicity. Dan Med Bull 1971;18:14-7.

5. Rufli T, Mumcuoglu Y. The hair follicle mites Demodex folliculorum and Demodex brevis: biology and medical importance. A review. Dermatologica 1981;162:1-11.

6. Temnikov VE. The peculiarity of immune status in rosacea. Nigniy Novgorod 1991;1:86-8.

7. Rather PA, Hassan I. Human demodex mite: the versatile mite of dermatological importance. Indian J Dermatol 2014;59:606.

8. Kito Y, Hashizume H, Tokura Y. Rosacealike demodicosis mimicking cutaneous lymphoma. Acta Derm Venereol 2012;92:169-70. 